\title{
AVALIAÇÃO DA INFLUÊNCIA DO CAMINHO DE LAMINAÇÃO NO TAMANHO DE GRÃO FINAL*
}

\author{
Paulo Jesus Aniceto ${ }^{1}$ \\ Gustavo Tressia² \\ Victor Caso Moreira² \\ Leandro de Ameida $^{3}$ \\ Marcos Tadeu Tavares Pacheco ${ }^{4}$
}

\section{Resumo}

Variações no tratamento termomecânico influenciam as propriedades dos metais e suas ligas. Em particular, na obtenção de chapas metálicas, a quantidade de passes de laminação e o grau de redução de cada etapa (caminho de laminação) influenciam na formação do estado deformado do material. Este estado, em conjunto com as condições de recozimento, determinará as propriedades finais do material. Este trabalho tem como objetivo estudar a influência do caminho de laminação no tamanho de grão final de dois materiais de diferentes energias de falha de empilhamento (EFE): cobre UNS C11000 e latão UNS C26000. As propriedades mecânicas das ligas escolhidas são em grande parte determinadas pelo tamanho de grão médio, que podem proporcionar aos materiais boa resistência mecânica aliada à boa ductilidade, condições desejadas em aplicações de estampagem. O estudo foi conduzido impondo uma redução total de $62 \%$ nas chapas utilizando-se duas condições diferentes: quatro e seis passes de laminação. $O$ recozimento foi comum para os dois casos e resultou em uma diferença significativa (6 $\mu \mathrm{m}$ ) entre os tamanhos de grão médios das amostras de latão, enquanto o mesmo não foi observado para o cobre. A distribuição obtida de tamanho de grão foi lognormal para todas as amostras, à exceção do latão laminado com quatro passes. Os resultados foram analisados qualitativamente à luz da estrutura dos materiais no estado encruado.

Palavras-chave: Cobre; Latão; Recozimento; Laminação.

\section{EVALUATION OF THE INFLUENCE OF THE ROLLING PATH ON THE FINAL GRAIN SIZE}

\begin{abstract}
Variations on the thermomechanical treatment of metals and alloys influence their properties. In particular, the rolling path of metal sheets influence the worked state of the material, that is, the number of passes and the deformation imposed in each pass. The worked state and the annealing parameters determine the mechanical and physical properties of the material. This work aims on the evaluation of the influence of the rolling path on the annealed grain size of two alloys of different stacking fault energies (SFE): copper UNS C11000 and brass UNS C26000. The mechanical properties of the chosen alloys are primarily determined by the mean grain size, which are responsible for a great behavior during deep drawing process. The strain imposed to the materials was $62 \%$ through two different conditions: four and six passes. The annealing conditions were the same for all materials. The final grain size of the brass samples turned out to be statistically different $(6 \mu \mathrm{m})$, which was not true for the copper samples. The grain size distribution was lognormal on all the samples, except for the brass sample rolled with four passes. The results were qualitatively analyzed based on the worked state of the materials.

Keywords: Copper; Brass; Annealing; Rolling.

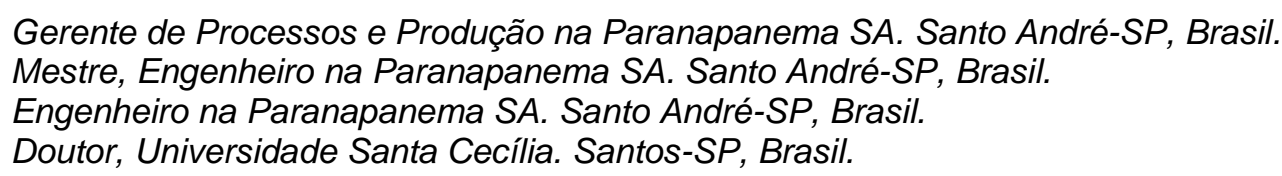

Gerente de Processos e Produção na Paranapanema SA. Santo André-SP, Brasil. Mestre, Engenheiro na Paranapanema SA. Santo André-SP, Brasil.

Engenheiro na Paranapanema SA. Santo André-SP, Brasil.

Doutor, Universidade Santa Cecília. Santos-SP, Brasil. 


\section{INTRODUÇÃO}

Desde os primórdios da metalurgia, sabe-se empiricamente que algumas variações do tratamento termomecânico influenciam de forma decisiva as propriedades dos metais e suas ligas. Os avanços da tecnologia e do conhecimento científico permitiram a compreensão da estrutura do material após deformações plásticas e das transformações que ocorrem durante o recozimento.

Em particular, na obtenção de chapas metálicas, a quantidade de passes de laminação e o grau de redução de cada etapa influenciam na formação do estado deformado do material. A natureza do metal ou liga, evidenciada na energia de falha de empilhamento (EFE), também exerce papel preponderante na formação do estado deformado, ou encruado. Este estado, em conjunto com as condições de recozimento, determinará as propriedades finais do metal ou liga.

Chapas e tiras de cobre e suas ligas são produzidas industrialmente incialmente por processos de fundição estática ou por fundição contínua. O processo de fundição estática é seguido da laminação a quente da placa e posteriormente laminação a frio. Já o processo de fundição contínua é seguido da laminação a frio diretamente, apresentando particularidades com relação à estrutura obtida e necessitando de outros cuidados. Em ambos os casos, a etapa final de produção frequentemente é o recozimento, proporcionando ao material maior ductilidade. As propriedades mecânicas, no entanto, serão definidas pelo tamanho médio de grão do material e pela distribuição de tamanho de grão.

O cobre e suas ligas permitem reduções totais por laminação superiores a $80 \%$ e reduções em torno de $40 \%$ entre passes. Essas características tornam possível processos produtivos excelentes, com baixo custo e rapidez, desde que seja realizado o entendimento dos impactos das etapas de manufatura das tiras e chapas no desempenho do material.

Os metais de maneira geral - o cobre e o latão inclusos -, quando deformados mecanicamente, absorvem parte da energia utilizada no processo de conformação e parte se dissipa em forma de calor. Padilha e Fulvio [1] afirmam que apenas 2 a 10\% da energia utilizada na deformação são absolvidas pelo metal e com isso ocorre o aumento da energia interna, sendo essa energia associada ao aumento de defeitos cristalinos, principalmente discordâncias. Quanto maior a deformação, maior será a densidade de discordância contida no metal (vide Tabela 1).

Souza [2] evidenciou em seu trabalho que o latão C26800 encrua mais rapidamente que o cobre C12000. Estudando amostras com redução de até $85 \%$, evidenciou um aumento de dureza em $80 \%$ para o latão e $60 \%$ para o cobre. O cobre apresentou um decréscimo da taxa de encruamento após 30\% de deformação, enquanto a taxa de encruamento do latão apresenta menor variação com a deformação. Essa diferença de evolução do encruamento é relacionada à diferença existente entre a EFE dos dois materiais, $62 \mathrm{~mJ} / \mathrm{m}^{2}$ para o cobre e $12 \mathrm{~mJ} / \mathrm{m}^{2}$ para o latão $(30 \%$ de zinco) [3]. Com menor EFE, a mobilidade das discordâncias no latão é reduzida, favorecendo o aumento da densidade deste defeito. 
Tabela 1. Densidade de discordâncias $\rho$ do cobre e latão obtidos pelo DRX [2].

\begin{tabular}{ccc}
\hline Amostras & $\begin{array}{c}\text { Cobre } \\
\text { (discordância/cm }\end{array}$ & $\begin{array}{c}\text { Latão } \\
\text { (discordância/cm }\end{array}$ \\
\hline recozida & $4,57 \times 10^{8}$ & $9,89 \times 10^{8}$ \\
\hline $10 \%$ reduzida & $1,86 \times 10^{10}$ & $5,43 \times 10^{10}$ \\
\hline $30 \%$ reduzida & $5,09 \times 10^{11}$ & $9,18 \times 10^{11}$ \\
\hline $50 \%$ reduzida & $5,87 \times 10^{11}$ & $6,34 \times 10^{12}$ \\
\hline $70 \%$ reduzida & $1,45 \times 10^{12}$ & $7,48 \times 10^{12}$ \\
\hline $85 \%$ reduzida & $1,43 \times 10^{12}$ & $7,58 \times 10^{12}$ \\
\hline
\end{tabular}

O arranjo de discordâncias e a ocorrência de heterogeneidades de deformação, como bandas de cisalhamento e bandas de transição, também são alterados pela EFE, pelo grau de redução e pela taxa de deformação $[4,5]$. A estrutura do material deformado é essencial para a recristalização do material, uma vez que a chamada nucleação deste processo ocorre preferencialmente em heterogeneidades de deformação [6, 7].

Assim, o presente estudo tem como objetivo avaliar a influência do número de passes na laminação a frio (caminho de laminação), e, portanto, da taxa de deformação, no tamanho de grão obtido após recozimento de dois materiais com diferentes EFE, o cobre e o latão.

\section{MATERIAIS E MÉTODOS}

As bobinas de partida de latão UNS C26000 e cobre UNS C11000 foram obtidas por fundição semi-contínua e laminação a quente. A composição química dos materiais, cedidos pela empresa PARANAPANEMA S/A é apresentada nas Tabelas 2 e 3. Em um laminador industrial do tipo quadruo, as bobinas foram laminadas conforme 0 plano de passes apresentado nas Tabelas 4 e 5 . O recozimento foi realizado em forno Mufla com temperatura de $490^{\circ} \mathrm{C}$ por um período de 180 minutos.

Tabela 2. Composição química das amostras da liga C26000.

\begin{tabular}{ccc}
\hline Elementos & Amostra 4 Passes & Amostra 6 Passes \\
\hline Cobre & 69,1500 & 69,2900 \\
\hline Chumbo & 0,0090 & 0,0058 \\
\hline Ferro & 0,0149 & 0,0099 \\
\hline Zinco & 30,8261 & 30,6943 \\
\hline
\end{tabular}

Tabela 3. Composição química das amostras da liga C11000.

\begin{tabular}{ccc}
\hline Elementos & $\begin{array}{c}\text { Amostra 4 } \\
\text { Passes }\end{array}$ & Amostra 6 Passes \\
\hline \% Cobre & 99,9865 & 99,9865 \\
\hline$\%$ Oxigênio & 0,0032 & 0,0030 \\
\hline$\% \sum$ Impurezas (Fe, P, Pb, Sn, Al e Zn) & 0,0103 & 0,0105 \\
\hline
\end{tabular}

As amostras para metalografia foram preparadas por lixamento com a lixa de carbeto de silício nas granulometrias \#320, \#400 e \#600. As amostras foram posteriormente polidas em um feltro com pasta de diamante de 6 e $1 \mu \mathrm{m}$ e atacadas quimicamente com a solução de Trióxido de Cromo. As imagens foram obtidas em microscópio vertical Olympus BX60M com câmera digital acoplada. Utilizou-se o software ImageJ para análise das imagens e determinação das áreas de cada grão. Para cada condição, foram analisados pelo menos 150 grãos, e o tamanho de grão 
médio foi determinado pela média das imagens analisada. A área dos grãos foi convertida em diâmetro médio equivalente pela suposição de um grão esférico.

Os resultados de análise de imagem foram tratados estatisticamente no software Minitab v.16, avaliando-se o tipo de distribuição dos resultados (Normal, Lognormal, de Weibull e Gamma) e gerando-se os histogramas de cada amostra.

Tabela 4. Plano de passe utilizado para o latão C26000.

\begin{tabular}{ccc}
\hline $\begin{array}{c}\text { No } \\
\text { Passe }\end{array}$ & $\begin{array}{c}\text { Espessura Latão } \\
\text { C26000 - 4 passes }\end{array}$ & $\begin{array}{c}\text { Espessura Latão } \\
\text { C26000 - 6 Passes }\end{array}$ \\
\hline 0 & 12,50 & 12,50 \\
\hline 1 & 10,00 & 10,50 \\
\hline 2 & 8,80 & 9,50 \\
\hline 3 & 6,20 & 8,50 \\
\hline 4 & 4,80 & 6,30 \\
\hline 5 & & 5,40 \\
\hline 6 & & 4,80 \\
\hline
\end{tabular}

Tabela 5. Plano de passe utilizado para o cobre C11000.

\begin{tabular}{ccc}
\hline $\begin{array}{c}\text { No } \\
\text { Passe }\end{array}$ & $\begin{array}{c}\text { Espessura Latão } \\
\mathbf{C 1 1 0 0 0}-\mathbf{4} \text { passes }\end{array}$ & $\begin{array}{c}\text { Espessura Latão } \\
\mathbf{C 1 1 0 0 0}-\mathbf{6} \text { Passes }\end{array}$ \\
\hline 0 & 12,50 & 12,5 \\
\hline 1 & 10,07 & 10,00 \\
\hline 2 & 8,87 & 9,50 \\
\hline 3 & 6,83 & 8,34 \\
\hline 4 & 4,78 & 6,80 \\
\hline 5 & & 5,42 \\
\hline 6 & & 4,80 \\
\hline
\end{tabular}

\section{RESULTADOS E DISCUSSÃO}

Diante dos dados de medição dos tamanhos de grão médio, realizou-se um teste de hipótese a fim de avaliar se estes são estatisticamente iguais. Para a realização dos testes foram assumidas três condições a serem seguidas:

- Condição inicial, $\mathrm{H}_{0}$, de que os tamanhos de grão são iguais;

- Nível de 5\% de significância;

- Desvios-padrão possuem a mesma ordem de grandeza.

O cálculo do $t$ de Student experimental (Eq. 1) se faz necessário para assim compará-lo com o valor crítico da distribuição $t$ de Student, extraído da Tabela A6.5 em Costa Neto (1977) [3]. Para a satisfação da condição $\mathrm{H}_{0}$, ou seja, para que os valores de tamanho de grão possam ser considerados estatisticamente iguais, 0 valor encontrado para o $t$ de Student experimental deve ser menor do que o valor crítico da distribuição $t$ de Student fornecido na tabela [3].

$$
\begin{array}{r}
t=\frac{\bar{x}_{1}-\bar{x}_{2}}{\left(s_{p} \cdot \sqrt{\left(\frac{1}{n_{1}+n_{2}}\right)}\right.} \\
S_{p}^{2}=\frac{\left[\left(n_{1}-1\right) * S_{1}\right]+\left[\left(n_{2}-1\right) * S_{2}\right]}{\left(n_{1}+n_{2}-2\right)}
\end{array}
$$

Sendo, $\mathrm{Sp}_{\mathrm{p}}^{2}$ a média ponderada das variâncias, $\mathrm{S}_{\mathrm{p}}$ a média ponderada dos desviospadrão e ni o número de amostragem da condição avaliada. 
Com os resultados disponibilizados na Tabela 6 , conclui-se que os valores de tamanho de grão para o material de latão são estatisticamente diferentes, pois o valor do $t$ encontrado é maior do que o valor crítico. Para o cobre, o valor do $t$ encontrado é menor do que o valor crítico, logo os tamanhos de grão são estatisticamente iguais.

Tabela 6. Valores para o teste de hipótese.

\section{Material Tamanho de grão [mm]}

\begin{tabular}{|c|c|c|c|c|c|c|c|c|}
\hline Liga & $\begin{array}{c}\text { № } \\
\text { passes }\end{array}$ & $\begin{array}{l}\text { Média } \\
\qquad(\bar{x})\end{array}$ & $\begin{array}{l}\text { Desvio } \\
\text { Padrão }\end{array}$ & $\begin{array}{c}\text { Variância } \\
\left(s^{2}\right)\end{array}$ & $\begin{array}{c}\text { Amostra } \\
\text { (n) }\end{array}$ & Sp2 & $\mathbf{t}$ & $\begin{array}{c}t \\
\text { crítico }\end{array}$ \\
\hline Latão & 4 & 25,4 & 1,5 & 2,2 & 3 & \multirow{2}{*}{1,4} & \multirow{2}{*}{6,1} & \multirow{2}{*}{2,1} \\
\hline Latão & 6 & 31,4 & 0,7 & 0,6 & 3 & & & \\
\hline Cobre & 4 & 27,0 & 2,2 & 4,8 & 3 & \multirow{2}{*}{2,9} & \multirow{2}{*}{1,7} & \multirow{2}{*}{2,1} \\
\hline Cobre & 6 & 24,6 & 1,0 & 1,0 & 3 & & & \\
\hline
\end{tabular}

O tamanho de grão médio do latão recozido após quatro passes de laminação é de $25,4 \mu \mathrm{m}$, em comparação ao valor de $31,4 \mu \mathrm{m}$ encontrado para a condição de 6 passes. A variação entre as condições é de $6,0 \mu \mathrm{m}$ e os desvios-padrão obtidos para as amostras é de 1,5 e 0,7 $\mu \mathrm{m}$, confirmando a diferença apresentada na análise do teste de hipótese. Por outro lado, para o cobre a diferença de tamanho de grão médio entre as duas condições é duas vezes menor, $2,4 \mu \mathrm{m}$, e os desvios-padrão são de 2,2 e 1,0 $\mu \mathrm{m}$, comprovando a igualdade entre os resultados identificada no teste de hipótese.

Os dados de tamanho de grão obtidos pelo software de análise de imagens ImageJ foram tratados estatisticamente no software Minitab. A distribuição dos dados de cada amostra foram testadas quanto às distribuições normal, lognormal, de Weibull e Gamma. As amostras de latão laminado com seis passes, cobre laminado com quatro passes e cobre laminado com seis passes apresentaram apenas distribuição lognormal em d (diâmetro equivalente dos grãos). As demais distribuições foram rejeitadas. A amostra de latão com quatro passes de laminação, por outro lado, não apresentou nenhum tipo de distribuição dos dados. A exposição dos dados em histogramas é apresentada na Figura 1, juntamente com as curvas teóricas da distribuição lognormal quando esta se adequou aos dados. 


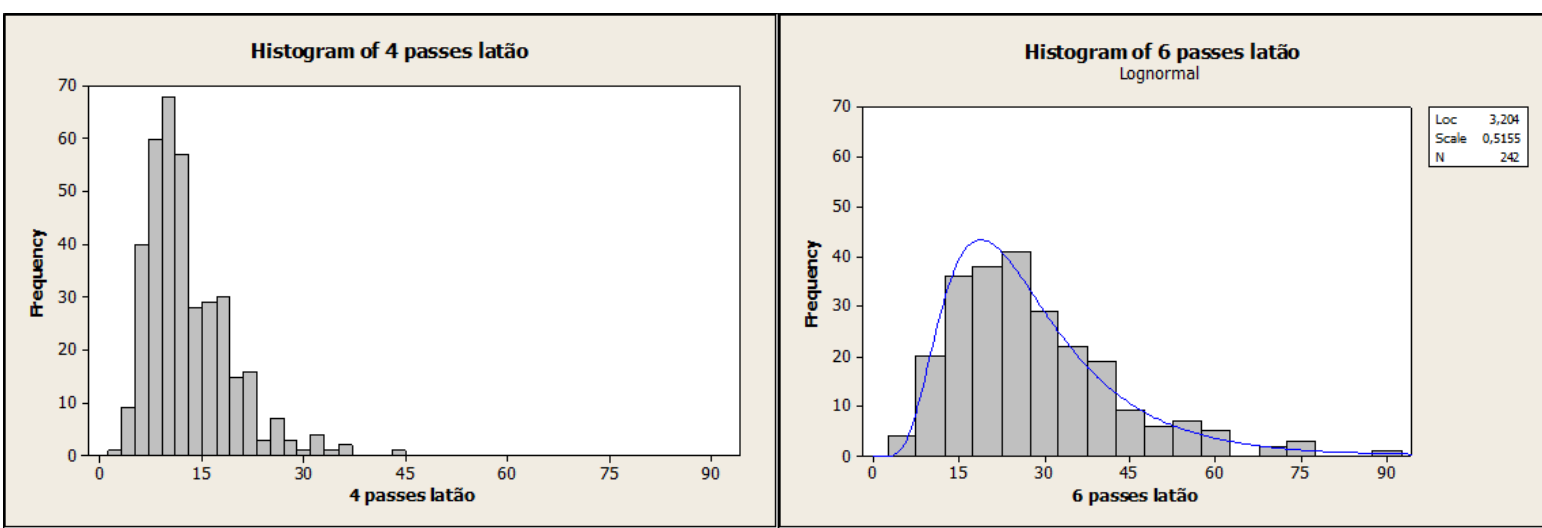

(a)

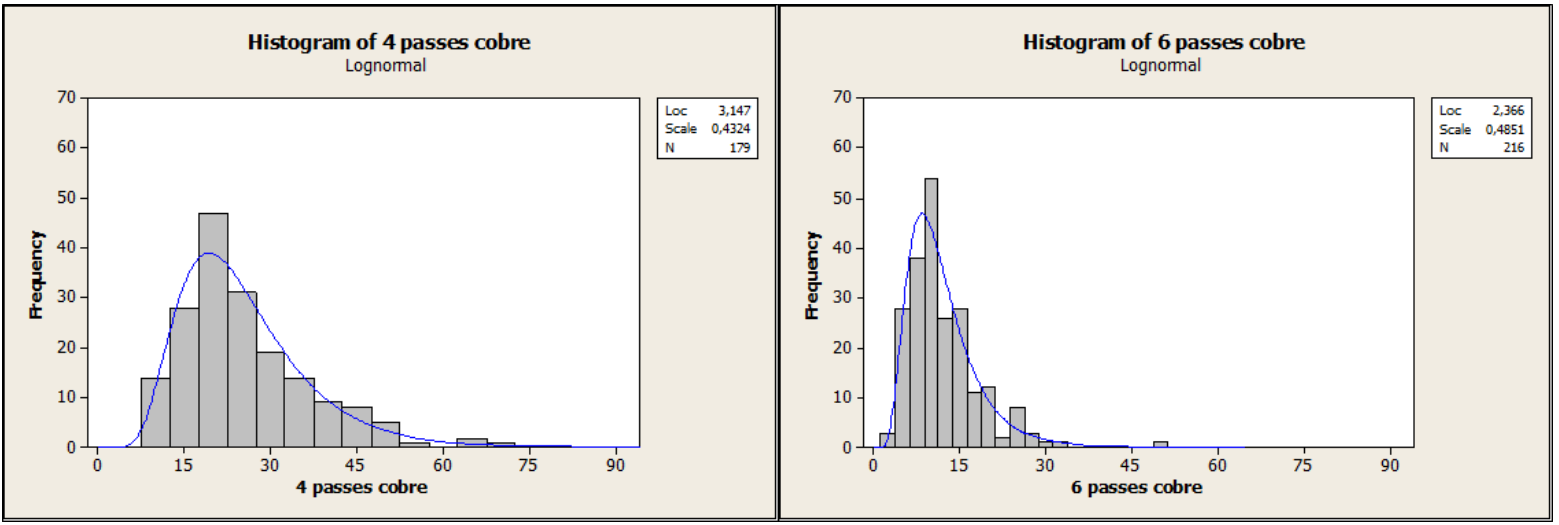

(b)

Figura 1. (a) Histogramas do latão 4 e 6 passes e (b) histogramas do cobre 4 e 6 passes.

O histograma do latão com quatro passes apresenta um comportamento distinto do latão com seis passes, pois este último apresenta um tamanho de grão médio superior e maior dispersão dos resultados, sendo evidenciado pelas frequências das classes no histograma. De outra forma, o cobre apresenta um comportamento oposto, ou seja, o material com quatro passes apresenta uma ligeira dispersão dos resultados, quando comparado ao cobre com seis passes de laminação, e ambos apresentam tamanho de grão médio similar.

As microestruturas das amostras são ilustradas na Figura 2. É possível observar maior tamanho de grão da amostra de latão com seis passes de laminação, quando comparada à amostra com quatro passes. O mesmo não pode ser observado para as amostras de cobre. Nota-se a importância da análise de imagens para observação de heterogeneidade microestrutural. 


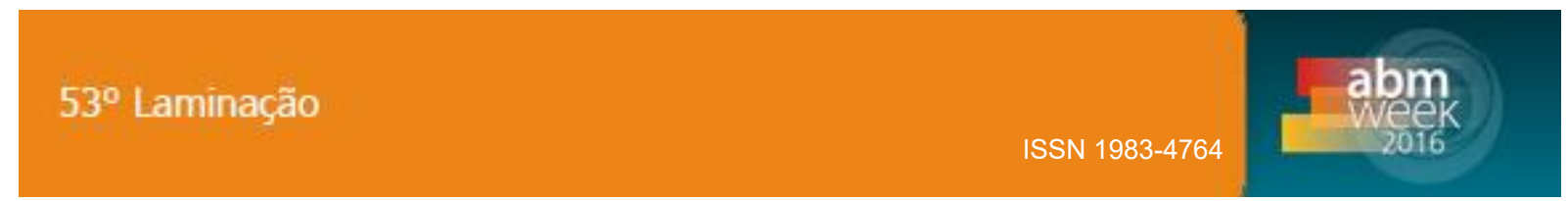

(a)

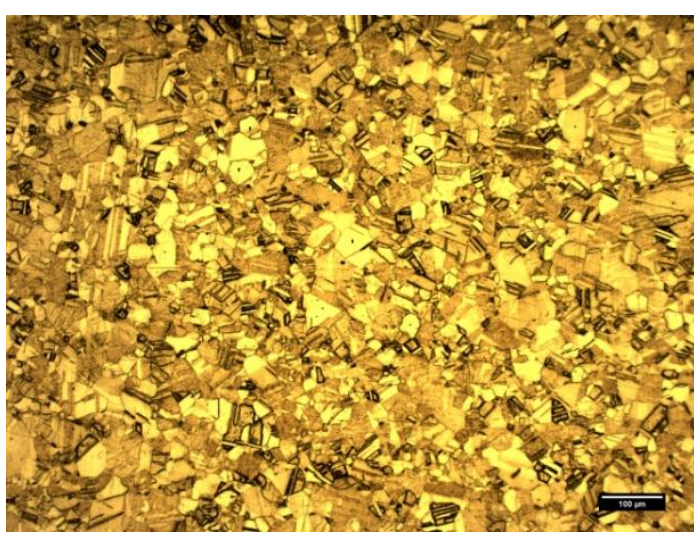

(c)

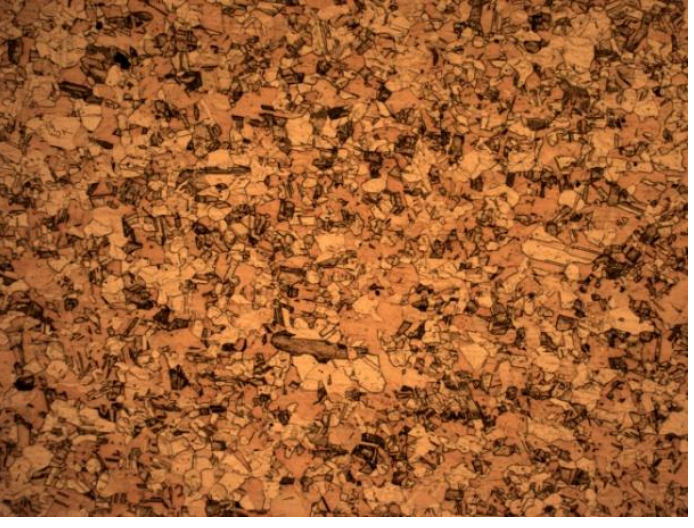

(b)

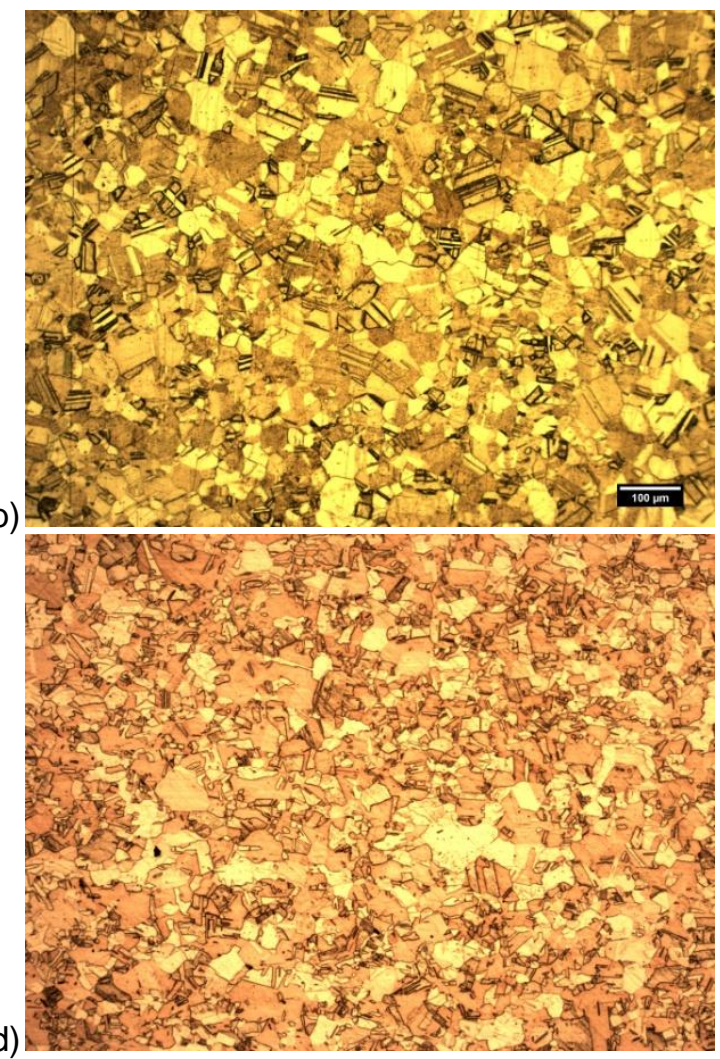

Figura 2. Microestrutura das amostras recozidas após laminação. (a) latão - 4 passes, (b) latão - 6 passes, (c) cobre -4 passes, e (d) cobre -6 passes.

A diferença de comportamento no recozimento das amostras de latão e de cobre em função da quantidade de passes de laminação pode ter origem nas diferenças microestruturais dos materiais deformados. Com menor EFE, o latão (12 $\left.\mathrm{mJ} / \mathrm{m}^{2}\right)$ apresenta uma distribuição de discordâncias mais uniforme que o cobre (62 mJ/m²). Porém, por outro lado, o aumento da taxa de deformação pode gerar mais heterogeneidades de deformação no latão, uma vez que a mobilidade das discordâncias é menor neste material. Assim, a amostra de latão com menos passes de laminação pode ter uma nucleação da recristalização mais intensa, dando origem a um tamanho de grão médio menor quando comparado à amostra com mais passes de laminação.

\section{CONCLUSÃO}

O número de passes (caminho de laminação) influencia o tamanho de grão médio após o recozimento para o latão C26000 (70/30). Para o cobre, essa variação não foi estatisticamente evidenciada. Além disso, observou-se maior heterogeneidade microestrutural nas amostras de latão laminadas com seis passes quando comparadas as com quatro passes. Essa tendência foi oposta para o cobre, embora em menor intensidade. Essas diferenças no comportamento do material podem estar associadas à diferença de EFE e consequente mobilidade de discordâncias entre as ligas estudadas, alterando assim a estrutura do material no estado encruado e consequentemente o tamanho de grão obtido após o recozimento. 


\section{REFERÊNCIAS}

1 Padilha AF, Siciliano JRF. Encruamento, recristalização, crescimento de grão e textura. 3 ed. São Paulo: Associação Brasileira de Metalurgia e Materiais, 2005. 232 p.

2 de Souza TG. Avaliação de densidade de discordâncias em cobre e latão $\alpha$ deformados por análise de largura de pico de DRX. Dissertação (mestrado) - Instituto Militar de Engenharia. 2014. 149 p.

3 Padilha, AF. Materiais de Engenharia. Curitiba: Hemus Livraria, Distribuidora e Editora SA. 2000. 343 p.

4 Humphreys FJ, Hatherly M. Recrystallization and related annealing phenomena. 2 ed. Oxford: Elsevier. 2004. 628 p.

5 Lee, WB, Duggan, BJ. Anneal Hardening in $\alpha$-brass. Metals Technology. 1983;10:8588.

6 Falleiros IGS, de Campos MF. Nucleação da recristalização. In: Tchiptschin AP. et al. (Eds). Textura e relações de orientação. 2 ed. São Paulo: IPEN. 2003. 480 p.

7 Hutchinson B. Nucleation of recrystallization. Scripta Metallurgica et Materialia. 1992;27:1471-1475. 